\title{
Firma INVITADA
}

\section{Il Ruolo delle Corti Supreme NELL'ATTUALE SISTEMA MULTILIVELLO*}

Pasquale GianNiti

Consigliere della Corte di cassazione italiana

Ormai da anni la dottrina, nel rilevare la rottura dell'unità sistemica dell'ordine normativo, ha proposto, per la comprensione delle relazioni tra le fonti del diritto, la metafora dell'arcipelago, da sostituire a quella, oramai superata e fuorviante, della piramide.

In un'epoca di moltiplicazione delle fonti del diritto, quale è la nostra, al centro del contesto ordinamentale (non sta più nella legge ordinaria, ma) stanno i princìpi costituzionali e le fonti sovranazionali, peraltro sempre più numerose e penetranti.

Tutto ciò pone in pericolo la generale esigenza di certezza del diritto, che è oggi sempre più avvertita non soltanto tra i giuristi, ma anche nel mondo dell'economia, della politica, dell'imprenditoria, dei comuni cittadini (nei rapporti tra loro e nei rapporti con la pubblica amministrazione).

L'esigenza di certezza del diritto è particolarmente avvertita nel settore dei diritti fondamentali: in questo settore l'ordinamento di qualsiasi Paese europeo, che sia nel contempo Parte contraente della Convenzione EDU e Stato Membro dell'Unione europea, si caratterizza oggi per la compresenza di 3 sistemi di salvaguardia, ciascuno con un proprio catalogo (la Costituzione nazionale, la Convenzione EDU, la Carta dei diritti fondamentali) e ciascuno con una propria corte di vertice (Corte costituzionale, Corte EDU, Corte di giustizia). Ai giorni nostri, ordinamento nazionale,

* Il testo costituisce riproduzione, con alcune aggiunte, dell'intervento pronunciato dall'autore in lingua spagnola in occasione del PhD Derecho, svoltosi presso la Universidad Complutense di Madrid nei giorni 27-28 settembre 2021.

Copyright: (C) Editorial Universidad de Sevilla. Este es un artículo de acceso abierto distribuido bajo los términos de la licencia de uso y distribución Creative Commons Reconocimiento-NoComercialSinObraDerivada 4.0 (CC BY-NC-ND 4.0) 
ordinamento convenzionale e ordinamento europeo sono ordinamenti che continuano ad essere tra loro distinti, ma che comunicano.

In questa prospettiva, occorre domandarsi se la nomofilachia delle corti supreme, nazionali ed europee, possa essere un antidoto all'odierna crisi della certezza del diritto.

Il vocabolo "nomofilachia", di origine greca, è composto dalle parole nomos (regola) e filachia (custode) e con esso si indicava, ai tempi dell'antica Grecia, la funzione svolta dai magistrati incaricati di custodire la stabilità della legislazione

Il vocabolo, dunque, ha radici antiche, ma non appartiene affatto alla "archeologia giuridica": anzi, la nomofilachia sta oggi divenendo un tema di teoria generale, in quanto investe problematiche di diverso ordine (che spaziano dal diritto europeo al diritto costituzionale e al diritto processuale) ed assume rilievo anche nel dibattito politico istituzionale.

Ai giorni nostri, la nomofilachia è un metodo di formazione del precedente. Le corti supreme, mediante la pronuncia di decisioni destinate a costituire "precedenti" per la soluzione di future controversie in casi simili o analoghi, sono chiamate a perseguire e realizzare la tendenziale uniformità nella interpretazione del diritto nazionale a tutela del generale principio di uguaglianza. In quanto tale, la nomofilachia costituisce un valore per l'intera collettività.

In Europa le corti supreme nazionali, pur presentando notevoli divergenze tra loro, hanno in comune il fatto di essere intese come le corti alle quali è istituzionalmente demandato il compito di curare lo sviluppo della nomofilachia: cioè di far emergere, tra le diverse possibili interpretazioni del diritto nazionale, quelle che meglio garantiscano la coerenza intrinseca ed estrinseca dei rispettivi ordinamenti.

Occorre ora chiedersi se quanto fin qui detto possa dirsi anche nell'attuale sistema multilivello in materia di diritti fondamentali.

In questa prospettiva occorre in primo luogo prendere atto del fatto che il contenuto della funzione nomofilattica della Corte di cassazione italiana sta cambiando e con esso sta cambiando anche la collocazione ordinamentale della Corte. Situazione analoga si sta verificando per le corti supreme nazionali di tutti gli altri Paesi membri.

Rispetto al nuovo contenuto, la nomofilachia della Corte ha cessato di essere collegata soltanto al diritto nazionale ed ha iniziato ad essere collegata anche al diritto sovranazionale, per cui la Corte oggi è chiamata a garantire l'uniforme interpretazione della legge nazionale, in conformità non soltanto dei disposti costituzionali, come interpretati dalla Corte costituzionale, ma anche dei disposti convenzionali, come interpretati dalla Corte EDU e, in conformità dei disposti europei, come interpretati dalla Corte di giustizia. La previsione dell'obbligo dell'interpretazione conforme ha sostanzialmente attribuito alla Corte di cassazione il compito di recepire gli effetti (diretti ed indiretti) della giurisprudenza delle corti supreme europee (Corte EDU e Corte di giustizia) in modo da prevenire la formazione di situazioni 
di contrasto tra l'ordinamento interno e gli ordinamenti sovranazionali (comunitario e convenzionale)

Rispetto alla nuova posizione, la Corte di cassazione è oggi inserita in una estesa e articolata rete di relazioni con le suddette corti supreme europee: in questo contesto, nuovo rispetto al passato, essa ha ceduto una quota della sua tradizionale supremazia, in quanto ha cessato di essere il punto finale della giurisdizione.

Questi due mutamenti (di contenuto della funzione e di collocazione ordinamentale), tuttavia, ben lungi dall'indebolire la funzione nomofilattica della Corte di cassazione italiana, l'ha rafforzata, in quanto la Corte, come d'altronde si verifica anche per le corti supreme nazionali degli Stati membri dell'Unione, ha assunto una dimensione sovranazionale, in quanto è divenuta interlocutrice delle corti supreme europee e, così facendo, partecipa ai processi di concretizzazione di diritti, destinati ad entrare nel circuito sovranazionale: la Corte si trova al crocevia di un sistema, che in materia di diritti fondamentali non è gerarchizzato, ma multilivello e che, proprio per tale ragione, innesca una circolarità di pronunce tra le corti dei diversi ordinamenti.

In definitiva: ai giorni nostri, in materia di diritti fondamentali, il compito della Corte di cassazione italiana, come quello delle altre corti supreme nazionali, è quello di governare il sistema multilivello delle fonti, offrendo ai giudici di merito orientamenti precisi e autorevoli in modo da prevenire la formazione di contrasti e di garantire la tendenziale stabilità dell'interpretazione del diritto nazionale su tutto il territorio dello Stato. Se questo è il ruolo ed il senso delle corti supreme in Europa oggi, si comprende agevolmente come esse possano essere un prezioso antidoto alla crisi della certezza del diritto, che oggi attraversano tutti i Paesi europei: la certezza del diritto - che nell' 800 , cioè nell'epoca del diritto dei codici, è stata perseguita mediante l'istituto della fattispecie - può essere perseguita ai nostri giorni (all'epoca del costituzionalismo moderno, in una epoca cioè nella quale il diritto è sempre più fatto di principi) mediante la nomofilachia delle corti supreme.

Se è certo che questo è il ruolo che anche la Corte di cassazione italiana, è chiamata a svolgere, occorre tuttavia chiedersi che cosa occorre fare per porre la Corte nelle condizioni di svolgere al meglio il suo ruolo.

E' doveroso precisare due incontrovertibili dati di fatto:

Primo dato: la Corte di cassazione italiana è assorbita ogni anno dall'esame di oltre 30 mila ricorsi civili e di oltre 50 mila ricorsi penali: in questo contesto è molto problematico, se non impossibile, mantenere una sufficiente uniformità di indirizzi giurisprudenziali all'interno della Corte e, ancor più problematico è manifestare all'esterno orientamenti giurisprudenziali, che siano dotati dell'univocità e della coerenza, richiesti dall'esercizio di un'autentica nomofilachia.

Secondo dato di fatto: il sistema italiano è storicamente basato sull'esistenza di più corti supreme: la Corte di cassazione, con giurisdizione in materia civile (in essa compresa 
la materia lavoristica e la materia tributaria) e in materia penale; il Consiglio di Stato con giurisdizione nel settore amministrativo e la Corte dei conti con giurisdizione in materia contabile. Senonché il settore dei diritti fondamentali è trasversale alle tre giurisdizioni, con la conseguenza che in tale settore è possibile la formazione di "nomofilachie" divergenti. Nell'ordinamento nazionale italiano si dà attualmente il seguente singolare paradosso: i giudici nazionali hanno strumenti di dialogo con i giudici sovranazionali, mentre, se appartengono ad ordini giurisdizionali diversi, non hanno strumenti per dialogare tra loro, neppure quando sono in gioco valori fondamentali comuni.

A fronte di questi due incontrovertibili dati di fatto si aprono, in sintesi, due prospettive:

a) in coerenza con le linee di tendenza proprie degli altri ordinamenti nazionali europei, occorrerebbe introdurre filtri al ricorso per cassazione in modo che la Corte decida soltanto questioni utili al fine del complessivo indirizzo della giurisprudenza (con conseguente superamento del problema dell'elevato numero dei ricorsi);

b) in coerenza con la posizione sovra-ordinata che l'ordinamento costituzionale italiano attribuisce alla Corte di cassazione rispetto alle altre corti supreme nazionali, il ruolo nomofilattico, già svolto dalla Corte nel settore dei diritti fondamentali, dovrebbe assumere rilevanza anche nei confronti del giudice amministrativo e del giudice contabile (con conseguente superamento del problema della formazione di nomofilachie divergenti in tale delicato settore della vita del Paese).

Si tratta di scelte, che potranno essere forse difficili, ma certo sono decisive per l'attuazione del principio della certezza del diritto e per il futuro della corte suprema nell'ordinamento nazionale italiano.

Il discorso a questo punto assume un respiro generale e attinge la crisi che il diritto attraversa nel mondo occidentale in una epoca storica caratterizzata da grandi trasformazioni.

Se è vero che sta cambiando il rapporto tra legislazione e giurisdizione, è anche vero che le corti supreme nazionali sono chiamate ad essere garanti della tendenziale uniforme interpretazione del diritto in vista della sua applicazione a tutela del generale principio di uguaglianza.

Riprendendo la felice metafora dell'arcipelago, elaborata da anni dalla dottrina, si potrebbe allora dire che la navigazione tra le tante isole del vigente arcipelago normativo è quanto mai difficile e pericolosa; ma sulle corti supreme grava la responsabilità di tracciare la rotta dei giudici territoriali, non con le ragioni dell'autorità, ma con l'autorità delle ragioni. 